\title{
PATENT PLUS: a blinded, randomised and extension study of riociguat plus sildenafil in pulmonary arterial hypertension
}

\author{
Nazzareno Galiè ${ }^{1}$, Katharina Müller ${ }^{2}$, Andrea-Viviana Scalise ${ }^{3}$ and \\ Ekkehard Grünig ${ }^{4}$
}

Affiliations: 'Dept of Experimental, Diagnostic, and Specialty Medicine (DIMES), University of Bologna, Bologna, Italy. ${ }^{2}$ Global Clinical Development, Bayer HealthCare Pharmaceuticals, Wuppertal, Germany. ${ }^{3}$ Global Clinical Development, Bayer Hispania, Barcelona, Spain. ${ }^{4}$ Center for Pulmonary Hypertension, Thorax Clinic, University Hospital Heidelberg, Heidelberg, Germany.

Correspondence: N. Galiè, DIMES, Bologna University Hospital, Via Massarenti 9, Bologna 40138, Italy. E-mail: nazzareno.galiedunibo.it

ABSTRACT PATENT PLUS evaluated the safety and efficacy of riociguat in combination with sildenafil in pulmonary arterial hypertension patients.

Patients receiving sildenafil ( $20 \mathrm{mg}$ three times daily) were randomised to placebo or riociguat (up to $2.5 \mathrm{mg}$ three times daily) for 12 weeks. The primary outcome was maximum change in supine systolic blood pressure (SBP) from baseline within $4 \mathrm{~h}$ of dosing. Secondary objectives comprised additional blood pressure, heart rate and exploratory efficacy variables, and safety. Patients could enter a long-term extension (LTE), where all patients received riociguat plus sildenafil.

There was no difference in maximum change in supine SBP from baseline within $4 \mathrm{~h}$ between the riociguat $(\mathrm{n}=12)$ (mean \pm SD baseline: $-20.2 \pm 15.3 \mathrm{mmHg}$; week 12: $-20.7 \pm 18.0 \mathrm{mmHg}$ ) and placebo groups $(\mathrm{n}=6)(-7.6 \pm 3.9$ and $-20.2 \pm 12.9 \mathrm{mmHg}$, respectively). Changes in standing SBP and supine or standing diastolic blood pressure were also not different. Combination therapy showed no favourable effects on exploratory clinical parameters, including haemodynamics and exercise capacity. In the LTE, there were high rates of discontinuation due to hypotension and three (18\%) deaths (not considered study drugrelated by the investigator).

There were potentially unfavourable safety signals with sildenafil plus riociguat and no evidence of a positive benefit/risk ratio. Concomitant use of riociguat with phosphodiesterase-5 inhibitors is therefore contraindicated.

@ERSpublications

Sildenafil+riociguat in PAH: no evidence of a positive benefit/risk ratio and potentially unfavourable safety signals http://ow.ly/Hbmfh

For editorial comments see Eur Respir J 2015; 45: 1211-1213 [DOI: 10.1183/09031936.00032715]

This article has supplementary material available from erj.ersjournals.com

Received: June 102014 | Accepted after revision: Dec 172014 | First published online: Feb 052015

Clinical trial: This study is registered at www.clinicaltrials.gov with identifier number NCT01179334.

Support statement: This study was supported by Bayer HealthCare Pharmaceuticals (Berlin, Germany). Editorial assistance was provided by Adelphi Communications Ltd (Bollington, UK), sponsored by Bayer HealthCare Pharmaceuticals. Funding information for this article has been deposited with FundRef.

Conflict of interest: Disclosures can be found alongside the online version of this article at erj.ersjournals.com

Copyright $\odot$ ERS 2015 


\section{Introduction}

Pulmonary arterial hypertension (PAH) is a chronic and life-threatening disease characterised by increased pulmonary vascular resistance (PVR) due to progressive vascular remodelling that can ultimately lead to right heart failure and death [1-3]. Current treatments for $\mathrm{PAH}$ include phosphodiesterase (PDE)-5 inhibitors, endothelin receptor antagonists (ERAs) and prostanoids [2]. However, despite the availability of these therapies for $\mathrm{PAH}$, there is still a significant unmet medical need in the management of this disorder and the mortality of patients with PAH remains high (1 year: $15 \%$; 3 years: $32 \%$ ) [4].

Pulmonary hypertension is associated with endothelial dysfunction, impaired nitric oxide (NO) synthesis and insufficient stimulation of the NO-soluble guanylate cyclase (sGC)-cyclic guanosine monophosphate (cGMP) pathway [5-7]. Riociguat is the first member of a novel class of therapeutics called sGC stimulators [6-9]. It has a dual mode of action, sensitising sGC to endogenous NO by stabilising NO-sGC binding and directly stimulating sGC via a different binding site, independently of NO, thereby leading to increased generation of cGMP [6, 7, 10]. In the pivotal phase III Pulmonary Arterial Hypertension Soluble Guanylate Cyclase-Stimulator Trial (PATENT)-1, riociguat was well tolerated, and improved exercise capacity and a range of secondary end-points in treatment-naïve patients with $\mathrm{PAH}$ and patients receiving pre-treatment with ERAs or nonintravenous prostanoids [11]. Consequently, riociguat was recently approved for the treatment of symptomatic PAH, offering a new specific drug therapy for PAH $[2,12]$.

PDE-5 inhibitors, such as sildenafil and tadalafil, prevent cGMP degradation but are dependent on cGMP synthesis and the presence of NO [1, 6]. PDE-5 inhibitors have been shown to be an effective therapy in patients with PAH. Therefore, we investigated the combination of sildenafil with riociguat, as an NO-independent stimulator of sGC, for possible synergistic effects in the treatment of patients with PAH, by increasing cGMP through stimulation of sGC and simultaneously inhibiting its breakdown. However, concomitant inhibition of PDE-5 and stimulation of sGC may also be poorly tolerated. Therefore, the PATENT PLUS study evaluated the effect of riociguat or placebo in combination with sildenafil on systolic blood pressure (SBP) over 12 weeks in patients with symptomatic PAH.

\section{Methods}

\section{Study subjects}

Patients with symptomatic PAH receiving stable ( $\geqslant 90$ days) sildenafil therapy (approved dose $20 \mathrm{mg}$ three times daily) were eligible for the study. Inclusion criteria included: age 18-75 years, 6-min walking distance $(6 \mathrm{MWD})>150 \mathrm{~m}, \mathrm{PVR}>300 \mathrm{dyn} \cdot \mathrm{s} \cdot \mathrm{cm}^{-5}$, mean pulmonary artery pressure $(\mathrm{mPAP}) \geqslant 25 \mathrm{mmHg}$, $\mathrm{SBP} \geqslant 95 \mathrm{mmHg}$ and heart rate $\leqslant 105$ beats $\mathrm{min}^{-1}$ in the first $2 \mathrm{~h}$ after taking sildenafil. Patients receiving treatment with other PDE-5 inhibitors, unspecific PDE inhibitors, ERAs, prostanoids or NO donors were not included, although supportive pulmonary hypertension therapies were permitted.

\section{Study design}

PATENT PLUS was a randomised, double-blind, placebo-controlled, multicentre study conducted in 11 centres across the Czech Republic, Germany, Italy, Spain and the UK from August 2010 to May 2013 (www.clinicaltrials.gov identifier number NCT01179334). The study assessed the effect of riociguat (1-2.5 mg three times daily), administered simultaneously with sildenafil (20 mg three times daily), on blood pressure up to $4 \mathrm{~h}$ after taking study medication in patients with symptomatic PAH. Patients were randomised 2:1 to receive riociguat or placebo using an interactive voice response system or an interactive web response system, in accordance with a computer-generated random code provided by Bayer Randomisation Management. Given the exploratory nature of this study, no formal sample size calculation was performed. The study was overseen by an independent and unblinded data monitoring committee (DMC); the DMC and the sponsor had the right to close the study at any time.

In accordance with previous clinical studies $[11,13]$, the dose of riociguat was individually adjusted from a starting dose of $1 \mathrm{mg}$ three times daily in $0.5-\mathrm{mg}$ increments every 2 weeks according to SBP and the appearance of symptoms during the 8-week dose-adjustment phase. However, dose reductions of riociguat for safety reasons were allowed at any point in time during the treatment phase. Patients then continued on their optimum dose for the 4-week maintenance phase (total treatment duration: 12 weeks).

Patients who completed the initial 12-week randomised study and tolerated the study medication well were offered the chance to participate in an open-label long-term extension (LTE), which assessed the long-term safety and tolerability of the riociguat/sildenafil combination. During the 8-week dose-adjustment phase of the LTE, riociguat patients from the main phase continued treatment with their individual "optimal" dose of riociguat with sham dose adjustment, whereas placebo patients from the initial study underwent blinded individual dose-adjustment of riociguat (1-2.5 $\mathrm{mg}$ three times daily), according to the same scheme used in the main phase of the study. Following the 8-week titration phase, patients received open-label therapy with their optimal dose for the remainder of the study. 
PATENT PLUS was carried out in accordance with Good Clinical Practice guidelines and the Declaration of Helsinki. The study protocol was approved by the ethics committees of all participating centres and all patients gave their written informed consent. The study was terminated by Bayer HealthCare Pharmaceuticals (Wuppertal, Germany) in 2012, in agreement with the principal investigator, based on discontinuations due to hypotensive events, serious adverse events (SAEs) and deaths in the noncontrolled LTE phase, together with the lack of signals for a favourable clinical effect in the randomised study phase. As a consequence, the second part of the PATENT PLUS study, designed to assess the combination of riociguat plus sildenafil at higher than approved doses, was cancelled.

\section{Procedures}

Blood pressure and heart rate profiles were first evaluated at the pre-treatment visit. Supine and standing blood pressure and heart rate were recorded 30 and $15 \mathrm{~min}$ prior to sildenafil administration, after sildenafil administration at 15, 30, 45 and $60 \mathrm{~min}$, and then half-hourly for the following $3 \mathrm{~h}$. Evaluation of blood pressure and heart rate following concomitant administration of sildenafil and riociguat or placebo on subsequent treatment visits followed a similar schedule. These time-points were chosen based on preliminary pharmacodynamic data, and were designed to capture the maximum change in blood pressure and heart rate following administration of study drug.

The overall study baseline SBP profile was the profile taken on study day 1 upon sildenafil administration before randomisation. After the first visit, patients were seen at weeks 2, 4, 6 and 8 during the titration phase, and at week 12 during the maintenance phase. The primary end-point was the maximum change in supine SBP from baseline within $4 \mathrm{~h}$ of dosing with riociguat or placebo. The maximum change from baseline at each visit was defined as the within-patient maximum decrease from baseline (or zero if baseline was lower than all subsequent SBP measurements in that profile) within $4 \mathrm{~h}$ after study drug administration. Each profile baseline was the last SBP recorded at and within 30 min before study drug administration.

Secondary variables included: maximum change in standing SBP, supine and standing diastolic blood pressure (DBP), and supine and standing HR from baseline within $4 \mathrm{~h}$ of study medication; area under effect curve for change from baseline in standing and supine SBP, DBP and heart rate within $4 \mathrm{~h}$ of study medication. Safety variables included adverse events, vital signs and laboratory parameters. Exploratory analysis of changes in 6MWD, World Health Organization (WHO) functional class, N-terminal prohormone of brain natriuretic peptide (NT-proBNP), Borg dyspnoea score, time to clinical worsening and haemodynamics (by right heart catheterisation) after 12 weeks was also undertaken.

During the LTE titration phase, patients had supine and standing blood pressure and heart rate recorded using the same schedule as during the initial 12-week study. For the remainder of the LTE, safety assessments took place every 3 months, without rigorous blood pressure assessment (standing blood pressure was measured before and $2-3 \mathrm{~h}$ after study drug intake during scheduled visits).

\section{Analysis}

All variables were analysed descriptively in this exploratory study and no formal statistical testing was planned. Data shown (mean \pm SD unless stated otherwise) were calculated from observed values at the respective visit and missing data were not imputed. Adverse events included all events that started from the time of administration of the first dose of study drug until 2 days after administration of the last dose.

\section{Results}

12-week randomised study

Patients

Of the 18 eligible patients who entered the initial 12-week randomised study, 12 were randomised and treated with riociguat, and six were randomised and treated with placebo (fig. 1).

Demographics were well balanced between groups at baseline; $50 \%$ of patients had idiopathic PAH and the majority were in WHO functional class II or III (89\%) (table 1). One (8\%) riociguat patient withdrew from the initial 12 -week randomised study due to an adverse event (blurred vision). At 12 weeks, $64 \%$ of patients were receiving riociguat $2.5 \mathrm{mg}$ three times daily, $27 \% 2 \mathrm{mg}$ three times daily and $9 \% 1.5 \mathrm{mg}$ three times daily.

\section{Blood pressure and heart rate}

There was no difference in the primary end-point, the maximum change in supine SBP from baseline within $4 \mathrm{~h}$ of study drug, between the riociguat and placebo groups (fig. 2). Mean \pm SD maximum change in supine SBP increased from $-20.2 \pm 15.3 \mathrm{mmHg}$ at overall study baseline $(\mathrm{n}=11)$ to $-20.7 \pm 18.0 \mathrm{mmHg}$ at week $12(\mathrm{n}=10)$ in the riociguat group, and from $-7.6 \pm 3.9 \mathrm{mmHg}(\mathrm{n}=5)$ to $-20.2 \pm 12.9 \mathrm{mmHg}(\mathrm{n}=5)$ in the placebo group. Mean absolute supine SBP was $130.2 \pm 17.6 \mathrm{mmHg}$ at baseline $(\mathrm{n}=12)$ and 


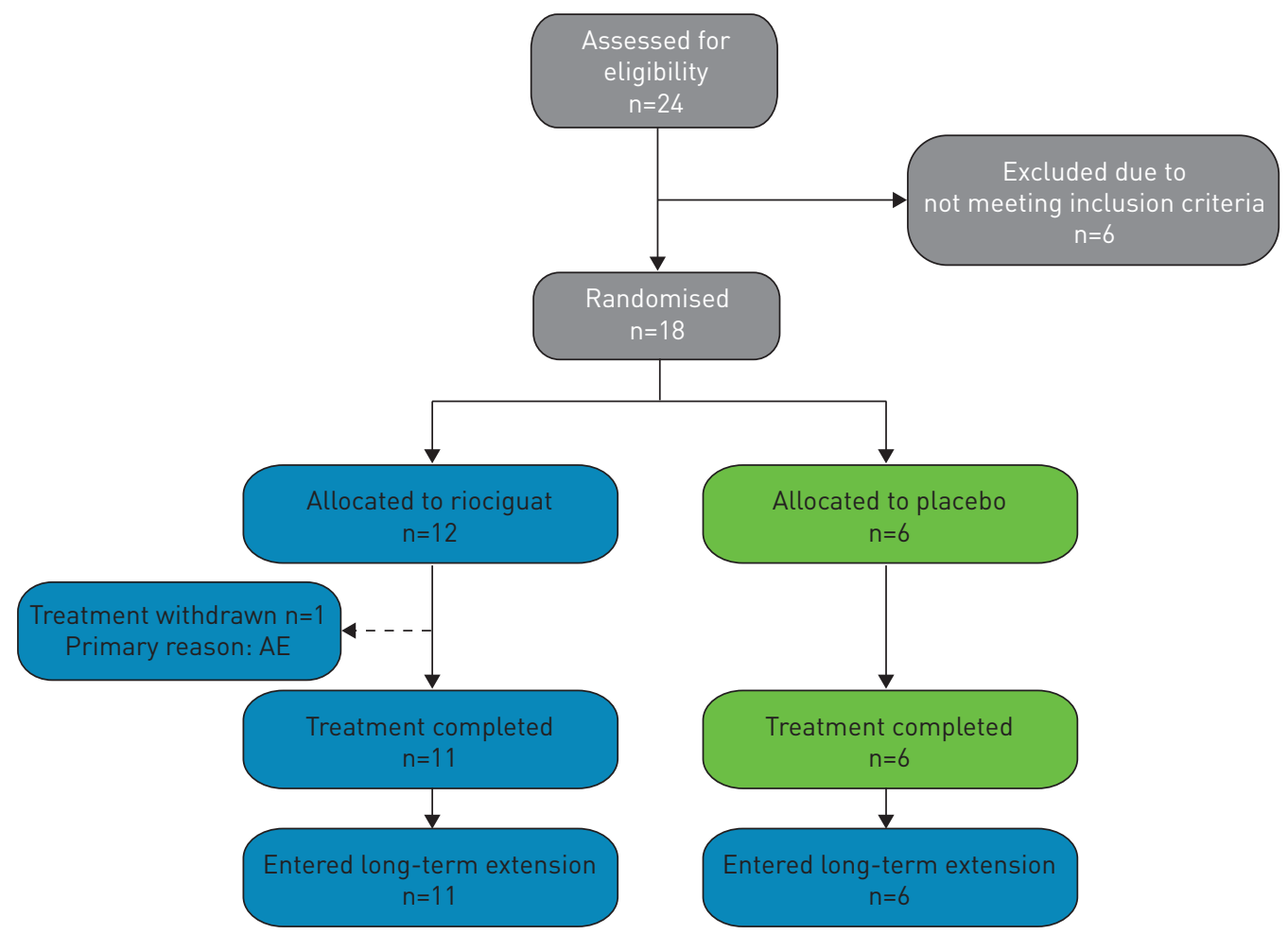

FIGURE 1 Patient disposition in PATENT PLUS. AE: adverse event.

117.1 $\pm 13.5 \mathrm{mmHg}$ at week $12(\mathrm{n}=11)$ in the riociguat group, compared with $127.0 \pm 17.9 \mathrm{mmHg}(\mathrm{n}=6)$ and 127.0 $\pm 10.3 \mathrm{mmHg}(\mathrm{n}=5)$, respectively, in the placebo group (fig. 3).

Mean maximum change in standing SBP from baseline to week 12 decreased from $-22.7 \pm 13.9$ to $-18.0 \pm 16.5 \mathrm{mmHg}$ in the riociguat group and increased from $-9.2 \pm 3.7$ to $-16.8 \pm 10.6 \mathrm{mmHg}$ in the placebo group (fig. 2). Mean absolute standing SBP was $124.1 \pm 17.4 \mathrm{mmHg}$ at baseline and $108.8 \pm 12.5 \mathrm{mmHg}$ at week 12 in the riociguat group, compared with $122.8 \pm 20.5$ and $119.0 \pm 9.8 \mathrm{mmHg}$, respectively, in the placebo group (fig. 3).

\section{TABLE 1 Baseline demographics}

\begin{tabular}{|c|c|c|}
\hline Parameter & Riociguat & Placebo \\
\hline Patients n & 12 & 6 \\
\hline Female & 8 (67) & $4(67)$ \\
\hline White race & $12(100)$ & $6(100)$ \\
\hline Age years & $58 \pm 11$ & $61 \pm 10$ \\
\hline \multicolumn{3}{|l|}{ Aetiology } \\
\hline Idiopathic PAH & $5(42)$ & $4(67)$ \\
\hline Connective tissue disease & $5(42)$ & $1(17)$ \\
\hline Portal hypertension & $1(8)$ & 0 \\
\hline Congenital heart disease & $1(8)$ & $1(17)$ \\
\hline \multicolumn{3}{|l|}{ WHO functional class } \\
\hline I & $1(8)$ & 0 \\
\hline II & $6(50)$ & $4(67)$ \\
\hline III & $4(33)$ & $2(33)$ \\
\hline IV & $1(8)$ & 0 \\
\hline Baseline $6 M W D \geqslant 320 \mathrm{~m}$ & 7 (58) & $6(100)$ \\
\hline Baseline PVR dyn $\cdot s \cdot \mathrm{cm}^{-5}$ & $573 \pm 241$ & $683 \pm 195$ \\
\hline
\end{tabular}

Data are presented as $\mathrm{n}(\%)$ or mean $\pm \mathrm{SD}$, unless otherwise stated. $\mathrm{PAH}$ : pulmonary arterial hypertension; WHO: World Health Organization; 6MWD: 6-min walking distance; PVR: pulmonary vascular resistance. 


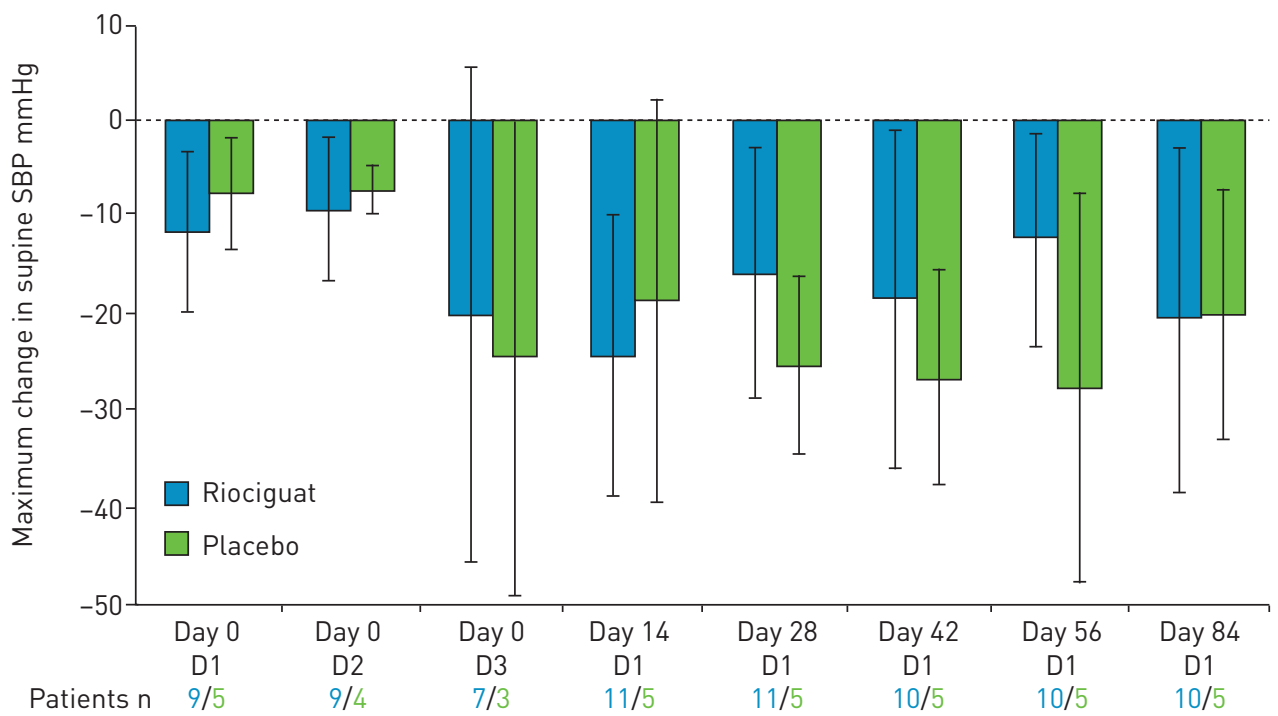

FIGURE 2 Mean maximum change in supine systolic blood pressure (SBP) (within $4 \mathrm{~h}$ from dosing) in the initial 12-week randomised study. Error bars represent standard deviations. D: dose.

There was no difference in the mean maximum change in supine or standing DBP between the riociguat and placebo groups. At week 12, mean maximum change in supine DBP was $-13.7 \pm 11.7 \mathrm{mmHg}$ in the riociguat group and $-13.8 \pm 12.9 \mathrm{mmHg}$ in the placebo group (mean absolute supine DBP at baseline: $73.1 \pm 8.6$ and $68.8 \pm 11.0 \mathrm{mmHg}$, respectively; week $12: 66.3 \pm 11.3$ and $64.6 \pm 9.7 \mathrm{mmHg}$, respectively) (fig. 3). Mean maximum change in standing DBP was $-14.4 \pm 10.6$ and $-14.2 \pm 10.0 \mathrm{mmHg}$, respectively (mean absolute standing DBP at baseline: $72.4 \pm 10.0$ and $68.0 \pm 13.9 \mathrm{mmHg}$, respectively; week $12: 62.1 \pm 9.7$ and $67.6 \pm 13.4 \mathrm{mmHg}$, respectively) (fig. 3). In an analysis of potential outliers, there were 32 low SBP $(<85 \mathrm{mmHg})$ or DBP $(<45 \mathrm{mmHg})$ events in the riociguat group $(\mathrm{n}=12)$ versus 24 in the placebo group $(\mathrm{n}=6)$.

At week 12, mean maximum change in supine heart rate was $5.9 \pm 7.1$ beats $\mathrm{min}^{-1}$ in the riociguat group $(\mathrm{n}=10)$ versus $9.6 \pm 5.5$ beats $\cdot \mathrm{min}^{-1}$ in the placebo group $(\mathrm{n}=5)$, and mean maximum change in standing
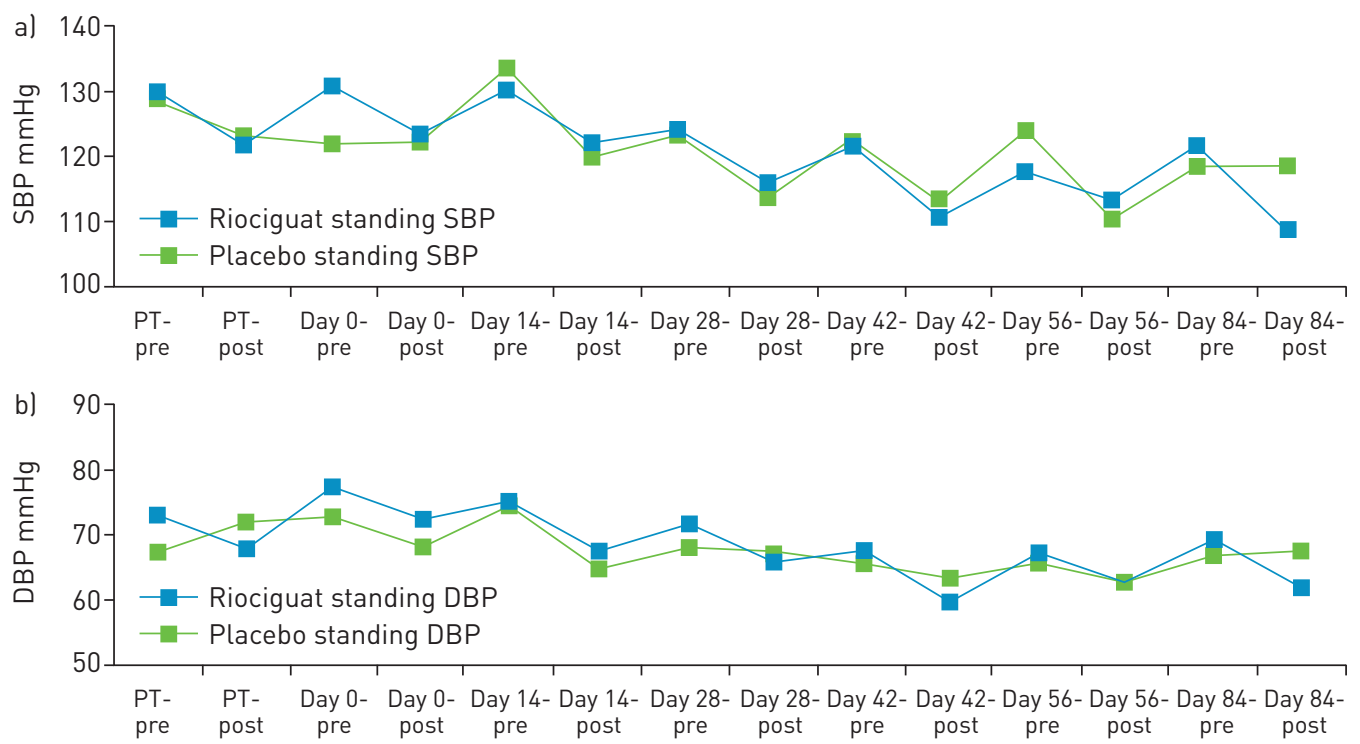

FIGURE 3 Changes in absolute systolic blood pressure (SBP) and diastolic blood pressure (DBP) over time in the initial 12 -week randomised study. At a particular time point, the values shown are means calculated from only those patients for whom data were available. Values shown on day 0 are pre- and post-dose 1. PT: pre-treatment; pre: pressure measured 30 min prior to study drug administration; post: pressure measured $4 \mathrm{~h}$ after study drug administration. 


\begin{tabular}{lcc} 
TABLE 2 Summary of safety in the initial $12-$ week randomised study & Placebo \\
& Riociguat & 6 \\
\hline Patients $\mathbf{n}$ & 12 & $4(67)$ \\
Any AE & $12(100)$ & $3(50)$ \\
Any study drug-related AE & $7(58)$ & 0 \\
AE-related deaths & 0 & 0 \\
Any SAE & $2(17)$ & 0 \\
Any study drug-related SAE & 0 & 0 \\
Discontinuation of study drug due to AEs & $1(8)$ & 0 \\
Discontinuation of study drug due to SAEs & 0 &
\end{tabular}

Data are presented as $\mathrm{n}(\%)$ unless otherwise stated. AE: adverse event; SAE: serious adverse event.

heart rate was $6.5 \pm 7.2$ and $10.4 \pm 6.5$ beats $\cdot \mathrm{min}^{-1}$, respectively. There were no between-group differences in the area under the effect curves in standing or supine SBP, DBP or heart rate (tables S1-S3).

Exploratory variables

There was no difference in change from baseline to week 12 between the riociguat and placebo groups in exploratory clinical parameters. Mean $6 \mathrm{MWD}$ increased by $7 \pm 48 \mathrm{~m}$ (baseline: $359 \pm 122 \mathrm{~m}$ ) in the riociguat group and by $30 \pm 56 \mathrm{~m}$ (baseline: $426 \pm 66 \mathrm{~m}$ ) in the placebo group, while WHO functional class improved/ stabilised in $18 \% / 82 \%$ of the riociguat group and $20 \% / 80 \%$ of the placebo group. NT-proBNP increased by $834 \pm 1858 \mathrm{pg} \cdot \mathrm{mL}^{-1}$ (baseline: $748 \pm 715 \mathrm{pg} \cdot \mathrm{mL}^{-1}$ ) in the riociguat group and decreased by $162 \pm 259 \mathrm{pg} \cdot \mathrm{mL}^{-1}$ (baseline: $278 \pm 330 \mathrm{pg} \cdot \mathrm{mL}^{-1}$ ) in the placebo group. No between-group differences in pulmonary haemodynamics, including PVR, systemic vascular resistance (SVR), mPAP and cardiac index, were seen (data not shown).

Safety

Adverse events were reported by 12 (100\%) riociguat patients and four (67\%) placebo patients (table 2). The most common adverse events are shown in table 3. Drug-related adverse events were reported by seven $(58 \%)$ riociguat patients and three $(50 \%)$ placebo patients.

Two riociguat patients reported SAEs (right ventricular failure and erysipelas); neither was considered to be drug-related by the investigator. The outcome of both events was reported as "recovered/resolved". One riociguat patient withdrew from the randomised phase of the study due to blurred vision (drug related). There were no deaths during the initial 12-week randomised study.

\begin{tabular}{lcc}
\hline TABLE 3 Most common adverse events in the initial & 12 -week randomised study & Placebo \\
\hline Patients $\mathbf{n}$ & Riociguat & 6 \\
Patients with $\geqslant 1$ AE & 12 & $4(67)$ \\
10 most common AEs & $12(100)$ & 0 \\
Hypokalaemia & & 0 \\
Peripheral oedema & $4(33)$ & $1(17)$ \\
Headache & $4(33)$ & $1(17)$ \\
Cough & $3(25)$ & $1(17)$ \\
Dizziness & $2(17)$ & $1(17)$ \\
Dyspepsia & $2(17)$ & 0 \\
Diarrhoea & $2(17)$ & 0 \\
Hypotension & $2(17)$ & 0 \\
Nasopharyngitis & $2(17)$ & 0 \\
Pyrexia & $2(17)$ & 0 \\
SAEs & $2(17)$ & 0 \\
Right heart decompensation & $1(8)$ & $1(8)$ \\
Erysipelas & &
\end{tabular}

Data are presented as $\mathrm{n}(\%)$ unless otherwise stated. AE: adverse event; SAE: serious adverse event. 


\section{Long-term extension}

Safety

All patients who completed the initial 12-week randomised study (riociguat, $n=11$; placebo, $n=6$ ) entered the LTE. The mean total treatment duration was 305 days. All 17 patients who entered the LTE reported adverse events, the most frequent being hypotension (41\%), dizziness $(24 \%)$, peripheral oedema (24\%), vomiting (24\%) and anaemia (24\%). Eight (47\%) patients reported a total of nine adverse events of special interest (table 4). Nine (53\%) patients reported 19 SAEs: hypotension ( $\mathrm{n}=2$; both drug related), acute right ventricular failure, angina pectoris, atrial fibrillation, cardiac arrest, chronic right ventricular failure, colitis (drug related), constipation, erysipelas, pneumonia, joint dislocation, subdural haematoma, intervertebral disc protrusion, loss of consciousness, syncope, acute respiratory failure, hypoxia and interstitial lung disease (all $n=1)$.

Six (35\%) patients withdrew from the LTE due to adverse events (table 4), which were all considered to be related to study drug by the investigator. There were three (18\%) deaths in the LTE (due to subdural haematoma following a fall, cardiac arrest and right heart failure), which were considered not to be drug related by the investigator (table 4). The patient who developed fatal subdural haematoma following a fall did not report experiencing dizziness, pre-syncope or syncope prior to the fall. However, in the investigator's opinion, systemic hypotension caused by riociguat prior to the fall cannot be excluded with certainty. There was no relationship between the riociguat dose administered and the incidence of discontinuations or adverse events of special interest.

In 2012, Bayer HealthCare Pharmaceuticals, in agreement with the principal investigator, decided to terminate the study based on hypotensive events, multiple discontinuations and three deaths in the LTE phase, together with the lack of signals for a favourable clinical effect in the randomised study phase. The DMC did not suggest prematurely terminating the trial at any time. The best possible treatment after discontinuation of riociguat was decided on an individual patient basis by the treating physician in accordance with current $\mathrm{PAH}$ treatment guidelines. Further data were collected during the 90-day follow-up period.

\section{Discussion}

The PATENT PLUS study was initiated to explore the tolerability and potentially favourable synergistic effects of riociguat, if added to a stable, approved dose of sildenafil in patients with symptomatic PAH. A safety outcome was selected as the primary end-point of the study, i.e. the maximum change in supine SBP from baseline within $4 \mathrm{~h}$ of riociguat administration on top of stable sildenafil treatment, compared with sildenafil alone. In the initial 12-week randomised study, addition of riociguat or placebo to sildenafil resulted in similar changes in blood pressure. However, the standing SBP after dosing at 12 weeks was approximately $10 \mathrm{mmHg}$ lower in the riociguat group compared with placebo (fig. 3). Furthermore, there were no clear benefits in exploratory efficacy variables, including 6MWD, haemodynamic variables and WHO functional class. Changes in NT-proBNP levels could not be interpreted due to differences in baseline values between the two groups. In the noncontrolled LTE phase, the combination of sildenafil and riociguat was associated with high rates of discontinuation due to hypotension and three deaths. Thus, it

TABLE 4 Summary of deaths, discontinuations and adverse events of special interest in the PATENT PLUS long-term extension (LTE) study

Deaths and discontinuations due to AEs and SAEs (53\%)

\section{3 deaths from SAEs $(18 \%)$}

Subdural haematoma after LTE month 6

Cardiac arrest after LTE month 9

Chronic right heart failure after LTE month 21

6 additional discontinuations due to AEs and SAEs $(35 \%)^{\#}$

3 AEs and 1 SAE of arterial hypotension at LTE days 14, 28, 42 and 84

$1 \mathrm{AE}$ of upper abdominal pain at LTE day 84

$1 \mathrm{AE}$ of vomiting at LTE month 15

Patients reporting AEs and SAEs of special interest (47\%)

1 SAE of syncope

1 SAE of loss of consciousness

5 AEs and 2 SAEs of hypotension ${ }^{\#}$

$4 \mathrm{AES}$ and $1 \mathrm{SAE}$ in the former riociguat group

$1 \mathrm{AE}$ and $1 \mathrm{SAE}$ in the former placebo group

Events were not considered related to study drug by the investigator, unless stated otherwise. AE: adverse event; SAE: serious adverse event. " : considered related to study drug by the investigator. 
was decided that the study should be discontinued (withdrawal of riociguat) in December 2012, with a 3-month follow-up. In totality, there were potentially unfavourable safety signals with sildenafil plus riociguat and no evidence of a positive benefit/risk ratio. Accordingly, concomitant use of riociguat with PDE-5 inhibitors (such as sildenafil, tadalafil and vardenafil) is contraindicated.

The underlying reason why no acute effect of combination therapy on blood pressure was evident in the randomised phase of the study yet discontinuations due to hypotension were frequent $(35 \%)$ in the LTE remains to be determined. The combination of a PDE-5 inhibitor and an sGC stimulator might result in an over-additive effect with a large increase in intracellular cGMP leading to pronounced vasodilation in the systemic circulation. The reduction in standing SBP in patients randomised to riociguat compared with placebo at 12 weeks may be explained by a potentially cumulative effect that is not directly demonstrated by data from the current study. In addition, we cannot rule out that changes in the dose of concomitant medication, such as diuretics, during the long-term extension may have contributed to the incidence of hypotension. This aspect and the role of other concomitant factors favouring clinical hypotension with these two combined vasodilators are difficult to explore further due to the current official contraindication.

Pharmacokinetic interactions are unlikely to explain the results observed in PATENT PLUS. Sildenafil is cleared predominantly by cytochrome P450 (CYP)3A (major route) and CYP2C9 (minor route) [14], whereas riociguat and its main metabolite are not inhibitors or inducers of major CYP isoforms or transporters in vitro at therapeutic plasma concentrations [15]. Conversely, riociguat is mainly cleared through metabolism by CYP1A1, CYP3A, CYP2C8 and CYP2J2 [16], and while sildenafil is a weak inhibitor of certain CYP isoforms (including CYP3A4), it is not expected to affect the pharmacokinetics of compounds that are substrates of these CYP enzymes at clinically relevant concentrations [14, 17]. Although pharmacokinetics were not investigated in the PATENT PLUS study, a small haemodynamic interaction study in seven patients with $\mathrm{PAH}$ found no pharmacokinetic interactions between riociguat and sildenafil (www.clinicaltrials.gov identifier NCT00680654).

While the benefit/risk ratio of combining riociguat and sildenafil is not favourable, data from the pivotal PATENT-1 study show that riociguat can provide additional clinical efficacy when added to ERAs and nonintravenous prostanoids [11]. In addition, these combinations were well tolerated, both in the randomised controlled phase of the study and during the LTE, PATENT-2 [18].

Direct comparisons between PATENT PLUS and PATENT require caution due to the different study designs and number of patients included. Nevertheless, $92 \%$ of riociguat $2.5 \mathrm{mg}$-maximum patients who were pretreated with ERAs or nonintravenous prostanoids in the 12-week PATENT-1 study $(\mathrm{n}=131)$ experienced a treatment-emergent adverse event and $15 \%$ of patients experienced an SAE (table 5) [11], versus 100\% and $17 \%$, respectively, in the present study. However, rates of discontinuation and drug-related SAEs were lower in patients in the PATENT-2 LTE study who received concomitant treatment with riociguat and other PAH therapies than in patients receiving riociguat plus sildenafil in the PATENT PLUS LTE [19]. Concomitant use of sildenafil and riociguat (mean treatment duration 305 days) resulted in higher rates of withdrawals

TABLE 5 Comparison between the safety of long-term riociguat in combination with sildenafil in the PATENT PLUS study with long-term riociguat in combination with prostanoids and endothelin receptor antagonists (ERAs) in PATENT-2

\begin{tabular}{lccc} 
& PATENT PLUS LTE & \multicolumn{2}{c}{ PATENT-2 $^{\pi}$} \\
\cline { 2 - 4 } & $\begin{array}{c}\text { Riociguat } \\
\text { +sildenafil }\end{array}$ & $\begin{array}{c}\text { Riociguat } \\
\text { +prostanoids }\end{array}$ & $\begin{array}{c}\text { Riociguat } \\
\text { +ERAs }\end{array}$ \\
\hline $\begin{array}{l}\text { Patients n } \\
\text { Mean treatment duration days }\end{array}$ & 17 & 23 & 161 \\
Maximum intensity of study drug-related AEs & 305 & 412 & 409 \\
$\quad$ Moderate & $4(24)$ & $4(17)$ & $41(25)$ \\
$\quad$ Severe & $2(12)$ & $3(13)$ & $10(6)$ \\
$\begin{array}{l}\text { AE-related deaths } \\
\text { Any SAE }\end{array}$ & $3(18)$ & $1(4)$ & $4(2)$ \\
Study drug-related SAE & $9(53)$ & $10(43)$ & $67(42)$ \\
Discontinuation of study drug due to AE & $3(18)$ & 0 & $13(8)$ \\
\end{tabular}

Data are presented as $n(\%)$ unless otherwise stated. LTE: long-term extension; AE: adverse event; SAE: serious adverse event. \#: cut-off, March 2013 (mean treatment duration 305 days); १: cut-off, April 2012 (mean treatment duration 409 days). 
due to adverse events ( $35 \%$ versus $8 \%$ and $17 \%$, respectively), drug-related SAEs ( $18 \%$ versus $8 \%$ and $0 \%$ ) and adverse event-related deaths ( $18 \%$ versus $2 \%$ and $4 \%)$ than riociguat plus ERAs $(\mathrm{n}=161$; mean treatment duration 409 days) or prostanoids ( $\mathrm{n}=23$; mean treatment duration 412 days) in PATENT-2 (previously unpublished data). Rates of hypotension ( $41 \%$ versus $7 \%)$ and discontinuations due to hypotension (24\% versus 0\%) were also higher in the PATENT PLUS LTE than in PATENT-2 [19]. Stronger stimulation of the NO-sGC-cGMP pathway with riociguat in combination with sildenafil is therefore not as well tolerated as riociguat monotherapy, or riociguat in combination with ERAs or prostanoids.

The limited information on efficacy from the small sample in PATENT PLUS did not indicate additional clinical benefit with this drug combination. Despite the high variability of data in this small exploratory study, greater increases from baseline in $6 \mathrm{MWD}$ and decreases from baseline in PVR were evident in the placebo group versus the riociguat group, despite similar changes in $\mathrm{mPAP}$, SVR and WHO functional class. Extrapolating the findings of small, exploratory clinical studies to larger populations should always be conducted with caution.

The PATENT PLUS study had several limitations. It was a relatively small study and no formal statistical testing was planned. Nevertheless, the clear safety signals due to multiple discontinuations in the LTE study prompted the cancellation of the planned additional study phases.

In conclusion, short-term addition of riociguat or placebo to sildenafil resulted in apparently similar changes in BP in the initial 12-week PATENT PLUS study, and there were no clear clinical benefits with riociguat/ sildenafil combination therapy in exploratory efficacy variables. In the PATENT PLUS LTE study, long-term therapy with riociguat in combination with sildenafil was associated with high rates of discontinuation due to hypotension, SAEs and deaths. Therefore, there were potentially unfavourable safety signals with sildenafil plus riociguat and no evidence of a positive benefit/risk ratio. Consequently, concomitant use of riociguat with PDE-5 inhibitors (such as sildenafil, tadalafil and vardenafil) is contraindicated.

\section{References}

1 Galie N, Hoeper MM, Humbert M, et al. Guidelines for the diagnosis and treatment of pulmonary hypertension: the Task Force for the Diagnosis and Treatment of Pulmonary Hypertension of the European Society of Cardiology (ESC) and the European Respiratory Society (ERS), endorsed by the International Society of Heart and Lung Transplantation (ISHLT). Eur Heart J 2009; 30: 2493-2537.

2 Galie N, Corris PA, Frost A, et al. Updated treatment algorithm of pulmonary arterial hypertension. J Am Coll Cardiol 2013; 62: D60-D72.

3 McLaughlin VV, Archer SL, Badesch DB, et al. ACCF/AHA 2009 expert consensus document on pulmonary hypertension: a report of the American College of Cardiology Foundation Task Force on Expert Consensus Documents and the American Heart Association: developed in collaboration with the American College of Chest Physicians, American Thoracic Society, Inc., and the Pulmonary Hypertension Association. Circulation 2009; 119: 2250-2294.

4 Benza RL, Miller DP, Barst RJ, et al. An evaluation of long-term survival from time of diagnosis in pulmonary arterial hypertension from the REVEAL Registry. Chest 2012; 142: 448-456.

5 Klinger JR, Abman SH, Gladwin MT. Nitric oxide deficiency and endothelial dysfunction in pulmonary arterial hypertension. Am J Respir Crit Care Med 2013; 188: 639-646.

6 Stasch JP, Pacher P, Evgenov OV. Soluble guanylate cyclase as an emerging therapeutic target in cardiopulmonary disease. Circulation 2010; 123: 2263-2273.

7 Stasch JP, Evgenov OV. Soluble guanylate cyclase stimulators in pulmonary hypertension. Handb Exp Pharmacol 2013; 218: 279-313.

8 Follmann M, Griebenow N, Hahn MG, et al. The chemistry and biology of soluble guanylate cyclase stimulators and activators. Angew Chem Int Ed Engl 2013; 52: 9442-9462.

9 Schermuly RT, Janssen W, Weissmann N, et al. Riociguat for the treatment of pulmonary hypertension. Expert Opin Investig Drugs 2011; 20: 567-576.

10 Stasch JP, Hobbs AJ. NO-independent, haem-dependent soluble guanylate cyclase stimulators. Handb Exp Pharmacol 2009; 277-308.

11 Ghofrani HA, Galiè N, Grimminger F, et al. Riociguat for the treatment of pulmonary arterial hypertension. N Engl J Med 2013; 369: 330-340.

12 Conole D, Scott LJ. Riociguat: first global approval. Drugs 2013; 73: 1967-1975.

13 Ghofrani HA, D'Armini AM, Grimminger F, et al. Riociguat for the treatment of chronic thromboembolic pulmonary hypertension. N Engl J Med 2013; 369: 319-329.

14 Pfizer. Revatio : US prescribing information. www.accessdata.fda.gov/drugsatfda_docs/label/2014/021845s011,02247 3s004,0203109s002lbl.pdf Date last updated: 2014.

15 Bayer HealthCare Pharmaceuticals. Adempas ${ }^{\circ}$. EU summary of product characteristics. www.ema.europa.eu/docs/en GB/document_library/EPAR__Product_Information/human/002737/WC500165034.pdf Date last updated: 2014.

16 Bayer HealthCare Pharmaceuticals. Adempas ${ }^{\star}$ US prescribing information. http://labeling.bayerhealthcare.com/ html/products/pi/Adempas_PI.pdf Date last updated: 2013.

17 Pfizer. Revatio: EU summary of product characteristics. www.ema.europa.eu/docs/en_GB/document_library/ EPAR_-_Product_Information/human/000638/WC500055840.pdf Date last updated: 2014.

18 Rubin LJ, Galiè N, Grimminger F, et al. Riociguat for the treatment of pulmonary arterial hypertension: a long-term extension study (PATENT-2). Eur Respir J 2015; 45: 1303-1313.

19 Rubin LJ, Galie N, Grimminger F, et al. Riociguat for the treatment of pulmonary arterial hypertension (PAH): a phase III long-term extension study (PATENT-2). Am J Respir Crit Care Med 2013; 187: A3531. 